Journal of Nuclear Materials

Manuscript Draft

Manuscript Number: JNM-D-14-00674

Title: Global erosion and deposition patterns in JET with the ITER-like Wall.

Article Type: Special Issue: PSI 2014 


\title{
Global erosion and deposition patterns in JET with the ITER-like Wall
}

\author{
A.Baron-Wiechec ${ }^{1}$, A.Widdowson ${ }^{1}$, E.Alves ${ }^{2}$, C.F.Ayres ${ }^{1}$, N. P. Barradas ${ }^{2}$, S.Brezinsek ${ }^{3}$, \\ J.P.Coad $^{1}$, N.Catarino ${ }^{2}$, K.Heinola ${ }^{5}$, J.Likonen ${ }^{4}$, G.F.Matthews ${ }^{1}$, M.Mayer ${ }^{6}$, P. Petersson ${ }^{7}$, \\ M.Rubel $^{7}$, W. van Renterghem ${ }^{8}$, I. Uytdenhouwen ${ }^{8}$ and JET-EFDA contributors ${ }^{9}$ \\ JET-EFDA, Culham Science Centre, OX14 3DB, Abingdon, UK \\ ${ }^{1}$ CCFE, Culham Science Centre, Abingdon, OX14 3DB, UK \\ ${ }^{2}$ Instituto Superior Técnico, Instituto de Plasmas e Fusão Nuclear,Universidade de Lisboa, Lisboa, Portugal \\ ${ }^{3}$ Association EURATOM-Forschungszentrum Jülich, IPP, D-52425, Jülich, Germany \\ ${ }^{4}$ Association EURATOM-TEKES, VTT, PO Box 1000, 02044 VTT, Espoo, Finland \\ ${ }^{5}$ Association EURATOM-TEKES, University of Helsinki, PO Box 64, 00014 University of Helsinki, Finland \\ ${ }^{6}$ Max-Planck-Institut für Plasmaphysik, 85748 Garching, Germany \\ ${ }^{7}$ Royal Institute of Technology, Assoc. EURATOM-VR, 10044 Stockholm, Sweden \\ ${ }^{8}$ SCK・CEN, the Belgian nuclear research centre, Boeretang 200, $2400 \mathrm{Mol}$, Belgium
}

${ }^{9}$ See the Appendix of F Romanelli et al., Proc. $24^{\text {th }}$ IAEA Fusion Energy Conference 2012 San Diego, USA

\begin{abstract}
A set of Be and W tiles removed after the first ITER-like Wall campaigns (JET-ILW) from 2011-2012 has been analysed. The results indicate that the primary erosion site is in the main chamber (Be) as in previous carbon campaigns (JET-C). In particular the limiters tiles near the mid-plane are eroded probably during the limiter phases of discharges. W is found at low concentrations on all plasma-facing surfaces of the vessel indicating deposition via plasma transport initially from the $\mathrm{W}$ divertor and from main chamber $\mathrm{W}$-coated tiles; there are also traces of Mo (used as an interlayer for these coatings). Deposited films in the inner divertor have a layered structure, and every layer is dominated by Be with some $\mathrm{W}$ and $\mathrm{O}$ content.
\end{abstract}


PACS: 52.40.Hf, 28.52.Fa

PSI-20 keywords: JET, ITER-like wall, plasma facing components, erosion, deposition, Ion beam analysis,

*Corresponding author address: Culham Centre for Fusion Energy, Culham Science Centre, Abingdon, OX14 3DB, UK

*Corresponding author E-mail: aleksandra.baron-wiechec@ccfe.ac.uk

Presenting author: Dr Aleksandra Baron-Wiechec

Presenting author email: aleksandra.baron-wiechec@,ccfe.ac.uk 


\section{Introduction}

Plasma wall interactions create some of the greatest challenges for the realisation of a fusion reactor. This is also true for ITER where tritium trapping due to implantation and codeposition and plasma pollution due to impurities migrating from PFCs to the plasma are major concerns. After over two decades of JET operation with a carbon wall, the ITER-like wall project at JET (JET-ILW) was initiated to explore plasma performance and plasma-wall interaction processes with a full metal wall: bulk beryllium $(\mathrm{Be}), \mathrm{Be}$-coated Inconel in the main chamber and bulk tungsten (W) or W-coated carbon fibre composites (CFC) in the divertor $[1,2]$. The transition to all metal PFC was an essential step to minimize hydrogen fuel retention. The first period of operation after installation of the new wall ran from September 2011 to July 2012 [3, 4]. An extensive post-mortem surface analysis program on PFC has been carried out after the ILW campaign and initial results were published elsewhere $[5,6,7]$. In this paper a more complete accounting of erosion and deposition regions is presented, with data from fifteen locations in the JET chamber cross-section as indicated in Fig 1, and more detailed characterization of the divertor deposition is now available.

\section{Experimental}

Be marker tiles at poloidal locations in the main JET chamber and W coated CFC marker tiles in the divertor have been installed as shown in Figure 1: The Be marker tiles have an interlayer of $\mathrm{Ni}$ with a top layer of $\sim 8 \mu \mathrm{m} \mathrm{Be}$, whilst the $\mathrm{W}$ coated CFE marker tiles have a Mo interlayer and a top layer of $\sim 4 \mu \mathrm{m} \mathrm{W}$ as described in $[3,8]$. The marker tiles were analysed prior to installation and after removal from the vessel by a set of ion beam analytical methods (IBA) to determine the extent of erosion/deposition. Ion beam analyses were carried out using a Van de Graaff accelerator at the IST/ITN in Lisbon. Three complementary techniques were used: nuclear reaction analysis (NRA), Rutherford Backscattering Spectrometry (RBS) and Particle Induced X-ray Emission (PIXE). NRA employed the ${ }^{9} \mathrm{Be}\left({ }^{3} \mathrm{He}, \mathrm{p}\right){ }^{11} \mathrm{~B},{ }^{12} \mathrm{C}\left({ }^{3} \mathrm{He}, \mathrm{p}\right){ }^{14} \mathrm{~N}$ 
and ${ }^{2} \mathrm{H}\left({ }^{3} \mathrm{He}, \mathrm{p}\right){ }^{4} \mathrm{He}$ reactions for detection of Be, $\mathrm{C}$ and ${ }^{2} \mathrm{H}$ using a $2.3 \mathrm{MeV} \mathrm{He}^{3}$ beam at normal incidence, with the detector positioned at $135^{\circ}$ to the direction of the incident beam. The beam spot diameter was $\sim 1 \mathrm{~mm}$. The chemical composition and thicknesses of the deposited layers were also analysed by RBS and PIXE using a $2.3 \mathrm{MeV}$ proton beam at a scattering angle of $150^{\circ}$. A $140 \mu \mathrm{m}$-thick Al film was placed in front of the detector to stop elastically-scattered particles, thereby providing almost background-free detection. To allow more accurate calculation of Be concentration in the deposits, an effort has been made to obtain the experimental cross-sections for backscattering of protons from $\mathrm{Be}$ and the $\mathrm{Be}\left({ }^{3} \mathrm{He}, \mathrm{p}\right) \mathrm{B}$ nuclear reaction specifically for these experiment conditions. The reaction crosssection required for data processing depends on the beam energy and geometry of the experiment (scattering angle) and the reaction cross-section value might change dramatically, which means that simple extrapolation of reaction cross-section obtained for different conditions may lead to over- or under- estimated results. Quantitative analysis has been carried out by using ${ }^{2} \mathrm{H}$ implanted $\mathrm{W}$ samples containing $34.7 \times 10^{15}{ }^{2} \mathrm{H}$ atoms $\mathrm{cm}^{-2}$ with an accuracy of $\sim 3 \%$ - further details can be found in [9]. Experimental data were processed using NDF [10] and/or SIMNRA [11] software to determine the chemical composition and thickness of deposits. IBA analysis is supported by extensive analysis of surface profile changes of the selected marker tiles using a tile profiler. A detailed description of the profiler setup and the method of the measurement can be found in [6]. Samples cut from selected divertor tiles were examined by scanning electron microscopy (SEM) using a JEOL type 6310 SEM with Energy Dispersive Spectrometry (EDS) using Noran Instruments equipment. The applied voltage was $15 \mathrm{keV}$ for Secondary Electron (SE) Back-Scattered Electron (BSE) viewing and EDS.

\section{Results}


In this section the material migration pattern is summarised for key areas of the main chamber and divertor. The implications are discussed in the next section.

\subsection{Deposition pattern in the main chamber}

\subsubsection{Outer poloidal limiters (OPL)}

The erosion/deposition at the outer poloidal limiters (OPL) tiles is illustrated by the analyses of tiles 4D3, 4D14 and 4D23 located as indicated (Fig. 1). Visual inspection of the OPL has been reported in $[3,6]$. At the centre of a tile near the midplane (4D14) the marker coating has been eroded and only bulk Be remains (Fig 2, region a). Profilometry indicates erosion of a minimum of $10 \mu \mathrm{m}$. IBA shows re-deposition of $\mathrm{Be}$ and $\mathrm{Ni}$ onto both left and right sides (Fig.2, region b). However, the right side of the tile is also characterised by some flaking of the coating, which limits the validity of the IBA analysis. Nevertheless based on IBA it can be concluded that where the coating remains it is a mixture of mainly Be with $\mathrm{Ni}$ and $\mathrm{W}$. Between the eroded area and the ends of the tile are transition regions where the surface is virtually as the original marker layer (Fig. 2, region c). Overall the analyses indicate net erosion over the tile, which if it were the same extent for the middle plane Tile 14 of each of the $8 \mathrm{OPL}$ in the vessel would amount to erosion of $5 \mathrm{~g}$ for just these tiles during the ILW campaign. Secondly, there is no indication from the RBS spectra of significant erosion or deposition on tiles 4D3 and 4D23 since the marker coating is still intact. The tiles are relatively clean, however there are traces of $\mathrm{W}, \mathrm{Ni}, \mathrm{Mo}, \mathrm{Cr}$, and $\mathrm{Fe}$ on the surface, and NRA shows some D retention. Finally, surface profilometry results confirm that any profile changes were below the detection limit $( \pm 5 \mu \mathrm{m})$, so these tiles do not contribute anything to the net erosion/deposition in the vessel during the ILW.

Tiles B and C placed between outer divertor tiles and the main chamber (Fig 1) were also analysed by means of IBA. These CFC tiles were coated with an approximately $10 \mu \mathrm{m}$ thick W coating prior to installation. There is no measurable change in profile of either tile (i.e. less 
than $5 \mu \mathrm{m}$ ), and NRA shows Be deposition (if any) is close to the spectrum background, not exceeding $1 \cdot 10^{15} \mathrm{at} / \mathrm{cm}^{2}$.

\subsubsection{Inner wall guard limiters (IWGL)}

Visual inspection of the Inner Wall Guard Limiters (IWGL) namely 2XR3, 2XR10 and 2XR19 located as indicated (Fig. 1) was been reported in [3, 6]. Surface profilometry and IBA analysis of marker tiles 2XR3, 2XR10 and 2XR19 has been completed recently. At the centre of tile 2XR10 the marker coating has been completely removed and only bulk Be remains, and profilometry indicates erosion of up to $60 \mu \mathrm{m}$. Towards the ends of the tile there is deposition of some Be and plasma impurities such as $\mathrm{W}$ and $\mathrm{Ni}, \mathrm{Mo}, \mathrm{Cr}, \mathrm{Fe}$ (identified by PIXE) typical for Inconel composition. At the very ends of the tile the composition reverts to the original marker composition. The erosion estimated rate from the mid-plane of the IWGL based on surface profilometry is approximately $2.3 \cdot 10^{19}$ atoms s${ }^{-1}[3]$. If the erosion/deposition pattern on this tile were repeated at position 10 on all twelve Be IWGL, there would have been a net erosion of $\sim 8 \mathrm{~g}$ during the ILW campaign.

The marker layers at the centre part of the top limiter 2XR19 are conserved so no measurable erosion has occurred, there are traces of $\mathrm{W}$ and Ni deposition on the tile. At the left-hand side of the centre of $2 \mathrm{XR} 3$ the Be marker top layer is about $15-25 \%$ thinner with respect to initial thickness, but at the same time there is some deposition of impurities in this region. There is a thick deposit (mainly Be with no more than $0.05 \%$ of $\mathrm{W}$ and $0.08 \% \mathrm{Ni}$ ) of around $7-10 \cdot 10^{18}$ at $/ \mathrm{cm}^{2}$ predominantly on the right side of $2 \mathrm{XR} 3$, as shown in Figure 3 and discussed in the next section. Thus on balance IWGL tiles in positions 3 and 19 make no significant contribution to erosion/deposition in the main chamber.

\subsubsection{Distribution of $W$ and Ni on inner and outer limiters and dump plates (DP)}


$\mathrm{W}$ and Ni impurities are present on all the tiles in the main chamber, but their concentrations can vary considerably as shown in the examples in Figure 3; Ni distributions are only shown where it is clear the $\mathrm{Ni}$ is present as a deposited impurity and is separate from the Ni marker interlayer., The amounts of $\mathrm{W}, \mathrm{Ni}$ and also traces of $\mathrm{Fe}, \mathrm{Cr}$, Ti and Mo were calculated by combining RBS and PIXE data, and the combined amount does not exceed $0.1 \%$ of the elemental composition of the deposit. $\mathrm{W}$ and $\mathrm{Ni}$ have been found on all three investigated OPL tiles, with a higher concentration on the left-hand side of 4D3 and 4D23 (Figs 3a and 3c). Deposition of W and Ni has slightly different pattern for the 4D14 (Fig 3b): In the central part of the tile the W concentration is below $1 \cdot 10^{15} \mathrm{at} / \mathrm{cm}^{2}$ and reaches maximum of approximately $30 \cdot 10^{15} \mathrm{at} / \mathrm{cm}^{2}$ at the location indicted by arrow in Fig.2. On the surface of the middle IWGL (2XR10) and the bottom (2XR3) (Figs 3e and 3f, respectively), the W surface peak signal (accompanied by Ni surface signal, not related to the underlying marker) is greater on the right-hand side of the limiter, whereas the W concentration is higher on the left-hand side of the 2XR19 (Fig 3d). Extensive melting along the poloidal ridge of the dump plate (DP) was found at all locations around the top of the machine and appears toroidally uniform in character. The melted ridge is closest to the plasma. Higher content of W has been found on the DP side where arc tracks are visible and is in the range from $5-40 \cdot 10^{15}$ at $/ \mathrm{cm}^{2}$ and $\mathrm{Ni}$ concentration varies from $20-$ $50 \cdot 10^{15} \mathrm{at} / \mathrm{cm}^{2}$.

\subsection{Deposition pattern in the divertor}

The results of a study of the deposition and erosion zones in the divertor, including $\mathrm{Be}$ concentration and also $\mathrm{C}$ and $\mathrm{O}$ concentration where it was possible to calculate have been reported $[3,5,7]$. The composition of the deposit consist mainly Be with traces of $\mathrm{W}, \mathrm{Ni}$, Mo, Cr and Fe. Only small amounts of Be have been found on Tile 3, 4, 6 and 7 (less that $1 \cdot 10^{18}$ at $/ \mathrm{cm}^{2}$ ), indeed SEM analysis suggest there may be some signs of erosion of the lower front part of Tile 1 (see diagram in Fig. 1) and the upper part of Tile 3. The thickest deposit has 
been found on the top (apron) of Tile 1 (generally $\sim 15 \mu \mathrm{m}$, with two points where it might be more than $17 \mu \mathrm{m}$ thick since this is the maximum accessible depth for RBS using $2.3 \mathrm{MeV}$ protons) and on the High Field Gap Closure (HFGC) tile (in the range from 3 to $8 \mu \mathrm{m}$ ); there is no data from surface profilometry from these areas. RBS spectra of all the deposits, but particularly the thick deposit on Tile 1 have proved difficult to fit with the NDF or SIMNRA software. The surfaces are known to be rough, but it was not possible to say to what extent the problems were related to the surface roughness and/or the chemical composition variability of the deposit. By fitting the spectra using a series of $\mathrm{Be}-\mathrm{W}$ layers with varying $\mathrm{Be} / \mathrm{W}$ ratio a relatively good agreement with the measured spectra is obtained and from this the integrated Be and $\mathrm{W}$ amounts inferred. However, it should be kept in mind that the data analysis assumes a flat surface. For the derived depth profiles roughness effects are interpreted as depth profiles of elements. Nevertheless, it has been already shown that in case of ambiguities between depth profiles and surface roughness the total amounts of elements are still correct even if the depth profiles may not reflect the sample structure correctly [12]. Optical microscopy of cross-sections shows that there are thick deposits on top of the original tile surface, and that the films have a multi-layered structure with some of the layers being less than $1 \mu \mathrm{m}$ thick [7]. Recent scanning electron microscopy (SEM) of sample cross-sections has brought some clarification for interpreting RBS spectra and confirmed a multi-layered structure of the deposited material, which is clearly observed on Tile 1, as shown in Fig. 4a. When back-scattered electrons (BSE) are imaged in the SEM, heavy elements such as W show up brightly, whereas light elements appear dark; there is very clearly contrast between the layers of the deposit indicating that a heavy element is mixed in with the deposits. Furthermore, SEM mass analysis indicates that $\mathrm{W}$ is the main element present in what IBA indicates is a predominantly Be film (Be is not detectable by SEM analysis). However, the SEM analysis can not resolve individual layers. Nevertheless, combining the information of the BSE microscopy, the SEM analysis and IBA, it can be concluded that the deposited 
material has a multi-layered structure consisting of an outer layer of pure $\mathrm{Be}$ and inner layer of mixed $\mathrm{Be}$ and $\mathrm{W}$ layers with $\mathrm{W} / \mathrm{Be}$ ratio: $0.05-0.08$. The oxygen concentration was not possible to evaluate directly in this work by IBA but it might be likely that the inner layer contains also a significant amount of oxygen (up to $10 \%$, based on NDF data fitting). The top part of Tile 1 revealed areas of non-uniformity of the deposit (small cracks), Fig. 4b, whereas the front parts of Tile 1, and Tiles 4 and 6 are characterized by a cauliflower-like morphology, as in Fig. 4c. The amount of material deposited on Tiles 4 and 6 was generally below $\sim 2.5 \cdot 10^{18} \mathrm{at} / \mathrm{cm}^{2}$, however areas of highier deposition $\left(\sim 6 \cdot 10^{18} \mathrm{at} / \mathrm{cm}^{2}\right.$ of Be over the $1 \mathrm{~mm}{ }^{2}$ beam area) were found on the slopping of these tiles. The deposit is not homogenous due to surface roughness of the CFC components and hence the $\mathrm{W}$ coating [13]. Whilst the thickness of the deposition on average is of the order of a hundred nanometers to $1 \mu \mathrm{m}$, but optical microscopy shows that in localized spots it can increase up to $5 \mu \mathrm{m}$ [7]. In the thickest part of the deposit on Tile 4 it was again found that it is built up by a multi-layer structure of different intensity in the BSE image, indicating a different composition of the layers (Fig. 4d). No changes in tile profile for Tiles 3, 4, 6, 7 or 8 were resolvable with the profilometer, so any net erosion/deposition is less than a few microns. For Tiles 3, 4 and 6 the small amount of deposition observed probably offsets any erosion (despite some indications of erosion at the top of Tile 3), but one can only set an upper limit on their contribution to overall deposition in the divertor. For Tiles 7 and 8 net erosion is more likely than net deposition (as was observed in the JET-C campaigns), but the global figure is likely to be small.

\section{Discussion and Conclusion}

The latest quantitative assessment of the overall material migration balance based on a combination of surface profilometry and accelerator based ion beam techniques is summarized in the Table, which also compares these values with those for the last JET carbon 
campaign taken from the literature. The results are consistent with the preliminary values given in reference [3] but are based on the larger amount of data now available including microscopy and analysis of inner wall cladding, hence the results are more detailed and accurate. The general picture based on marker analysis and surface profilometry is that the major erosion areas are the central part of the Be IWGLs, with a lesser erosion area at the centre of the OPL, subject to the strong caveats that tiles from only a limited selection of poloidal locations were removed, and no check was made on toroidal uniformity other than the detailed visual examination of every tile in the vessel. The rate of erosion at the IWGL during the limiter phases of ILW operations has been calculated to be similar to that during the previous carbon campaign [3]. It is expected that erosion during the limiter phases will be balanced by re-deposition on limiter surfaces deeper into the scrape-off layer (SOL), but despite more measurements this balance has not been demonstrated due to the limited number of tile samples, surface roughness and/or flaking of coating in deposition areas [1,2]. During the divertor phase of each discharge erosion is probably dominated by charge exchange neutral $(\mathrm{CXN})$ bombardment of the main chamber wall, and is followed by migration along the SOL to the inner divertor. This is evidenced by erosion measurements at the Inner Wall Cladding (IWC) tiles that line the vessel wall between the IWGL [14] which suggest that the IWC may account for a significant fraction of the Be deposition found in the divertor. Some contribution may also be expected from re-erosion of the deposits on the IWGL, which may have a higher erosion yield than the bulk material. Melting of Be tiles occurred at Dump Plate tiles and also at some castellation edges on IWGL tiles. Furthermore, there is $\mathrm{W}$ found on the surface of all tiles in the JET vessel, including tiles that were pure Be, and the amounts at the surface are of the same order of magnitude everywhere, from Dump Plates at the top of the vessel to divertor tiles at the bottom, and even to the louvre clips in shadowed areas. It seems unlikely that the divertor was the major source of the W. Furthermore, transient impurity events involving tungsten are relatively common [15]. It seems likely, therefore that the 
source of the $\mathrm{W}$ and Mo is from small particles of $\mathrm{W}$ coating (which has a Mo interlayer) coming off coatings in the main chamber; there are significant areas of W-coated CFC in the main chamber as well as in the divertor, such as wall protection in neutral beam shine-through areas, mushroom limiters at the top of the vessel and diagnostic protection covers. There are also many areas where widespread arcing has occurred that are visible on Be tiles: such arcing may also be widespread on W-coated CFC tiles but would not be easily visible, however could easily dislodge coating asperities from these rough surfaces. Deposition at the inner divertor is concentrated on the HFGC tiles and the inboard parts of Tile 1. This pattern is more restricted than during the previous JET carbon campaigns, when deposition extended over all Tiles 1, 3, 4 and 6 as well as structures at the corners of the divertor. This may be partly due to the more limited range of plasma shapes employed during the ILW operations. However, the largest difference is probably the lack of strong chemical sputtering which both allowed low energy neutrals to efficiently sputter the carbon main chamber walls during the divertor phase and to re-erode the carbon deposited in the divertor, thus enabling long range migration within the divertor.

\section{Acknowledgement}

This work was partly funded by the RCUK Energy Programme [grant number EP/I501045] and by the European Union Horizon 2020 research and innovation programme. The work was also part-funded by the European Communities under the contract of Association between EURATOM/CCFE within the framework of the European Fusion Development Agreement. The views and opinions expressed herein do not necessarily reflect those of the European Commission. 


\section{Reference}

[1] G.F. Matthews et al. Phys. Scr. (2011) 014001

[2] C. Giroud et al. Nucl. Fusion 53 (2013) 113025

[3] A. Widdowson et al. 2014 Phys. Scr. T159 (2014) 014010

[4] G.F. Matthews et al. J. Nucl. Mater. 438 (2013) S2-S10

[5] J. Likonen et al. Phys. Scr. T159 (2014) 014016

[6] K. Heinola et al. Phys. Scr. T159 (2014) 014013

[7] J.P. Coad et al. 2014 Phys. Scr. T159 (2014) 014012

[8] K. Heinola et al. Phys. Scr. T128 (2007) 91-95

[9] K. Heinola et al. this conference

[10] N.P. Barradas, C. Jeynes, Nucl. Instr. Meth. B 266 (2008) 1875

[11] M. Mayer, SIMNRA User Guide, Report IPP 9/113, Max-Planck-Institut für Plasmaphysik, Garching, Germany, 1997

[12] M. Mayer et al. Nucl. Instr. Meth. B 269 (2011) 3006

[13] M. Mayer et al. V 2007 J. Nucl. Mater. 363-5 101-6

[14] S. Krat et al. J. Nucl. Mater. 456 (2015) 106-110

[15] M. Sertoli et al. this conference

[16] M. Mayer et al. J. Nucl. Mater. 438 (2013) S780-S783

[17] J.P. Coad, J Likonen et al. this conference 


\section{Figure caption}

Fig.1. (a) Poloidal cross-section of the JET vessel with the location of the investigated tiles indicated (b) Diagram of Tile 1 showing the regions referred to in the text.

Fig.2. Photo of OPL (4D14) after ILW campaigns 2011-2 and the marker coating evaluation. A central erosion zone (indicated by a), a left and right wing deposition zone (indicated by b), and a middle part where the marker starts appearing (indicated by c). A region of flaking film is also indicated.

Fig.3. W and (in some cases) Ni impurity concentrations across the OPL and IWGL tiles analysed: a) OPL tile 4D23, b) OPL tile 4D14, c) OPL tile 4D3, d) IWGL tile 2XR19, e) IWGL tile 2XR10, f) IWGL tile 2XR3

Fig.4.(a) BSE image of a cross-section of the top part (apron) of Tile 1 (the deposited layer has separated from the tile surface during the mounting process), (b) BSE image of a top view of the of the Tile 1 apron surface, (c) BSE image of a cross-section of the sloped part of Tile 4 near the strike point showing deposit in the valleys, (d) BSE image of a top view of the Tile 4 surface with characteristic cauliflower morphology

\section{Table caption}

\section{Table 1}

Quantitative assessment of the net erosion/deposition at the various marker tiles analysed (extrapolated to all the toroidal tiles at the same poloidal location) following the JET-ILW campaign 2011-2, and comparison with the data (from the literature) following the JET-C campaign 2007-9. 


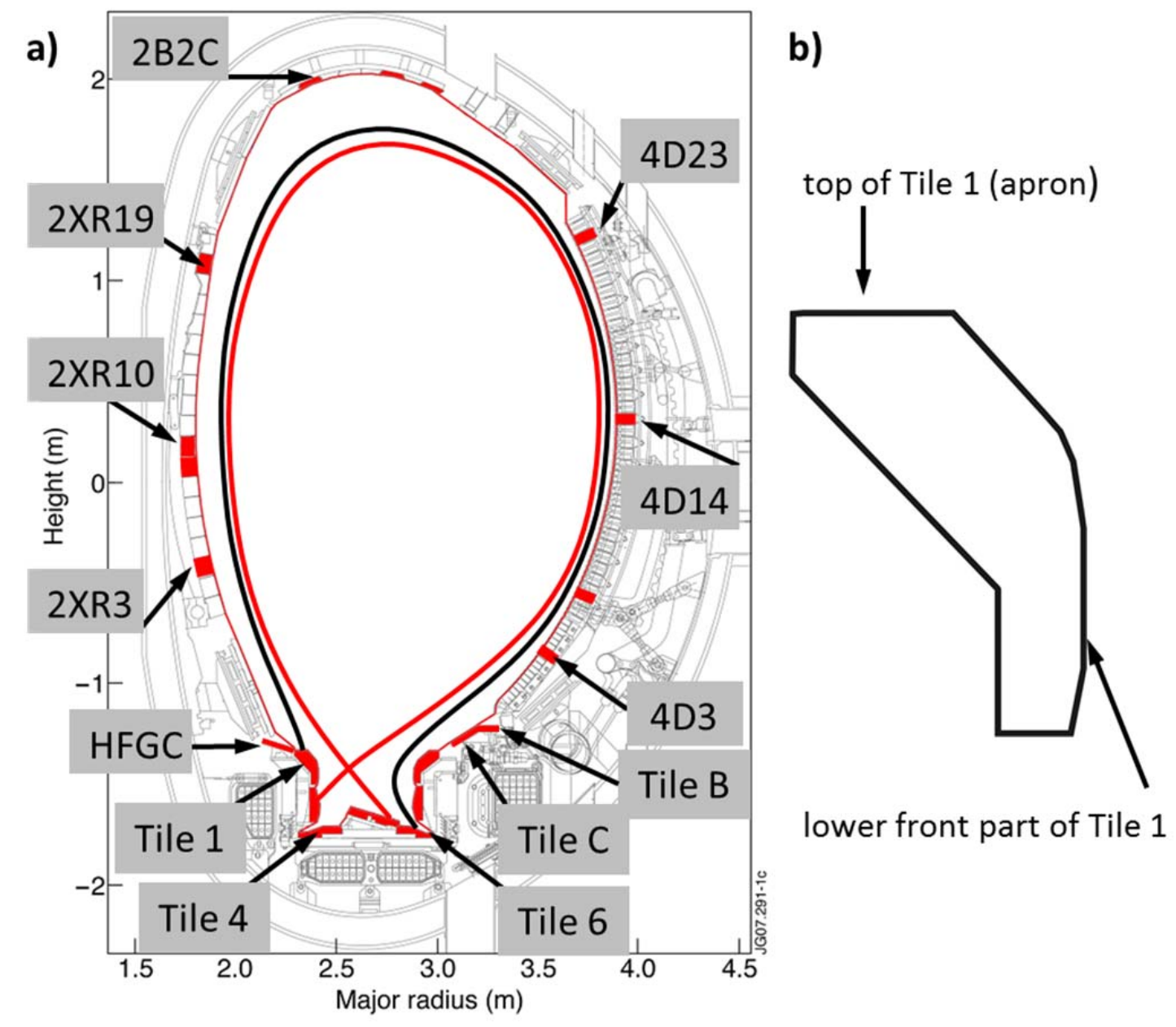

Fig.1. 


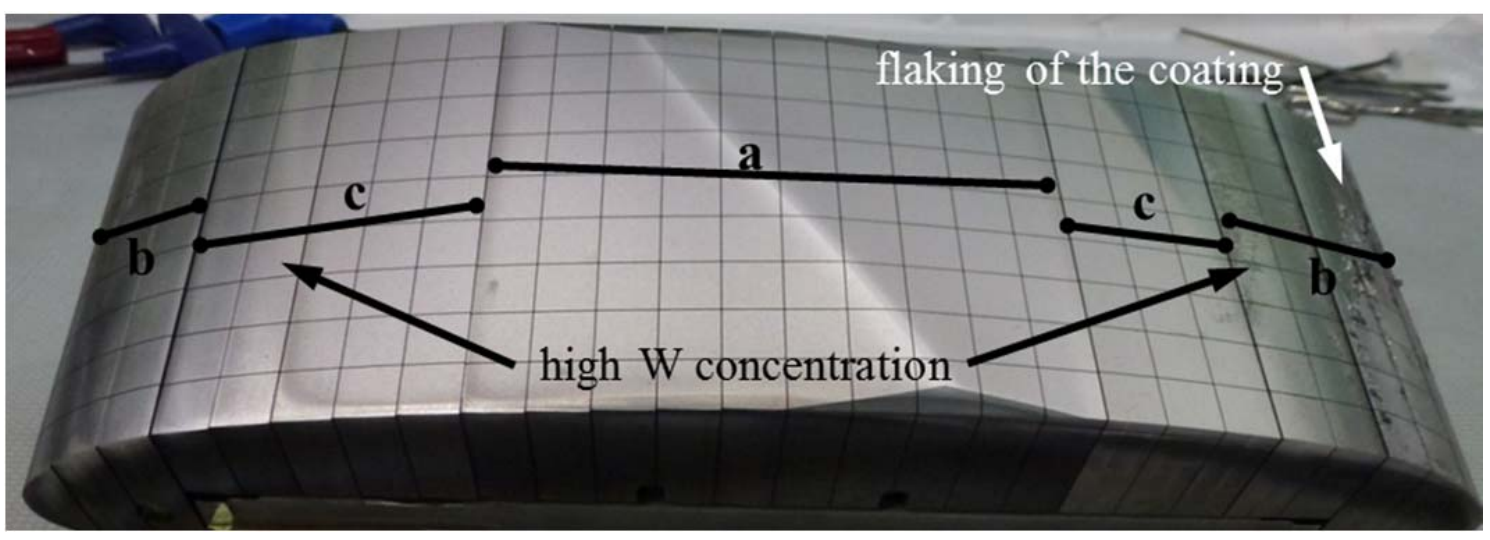

Fig.2.

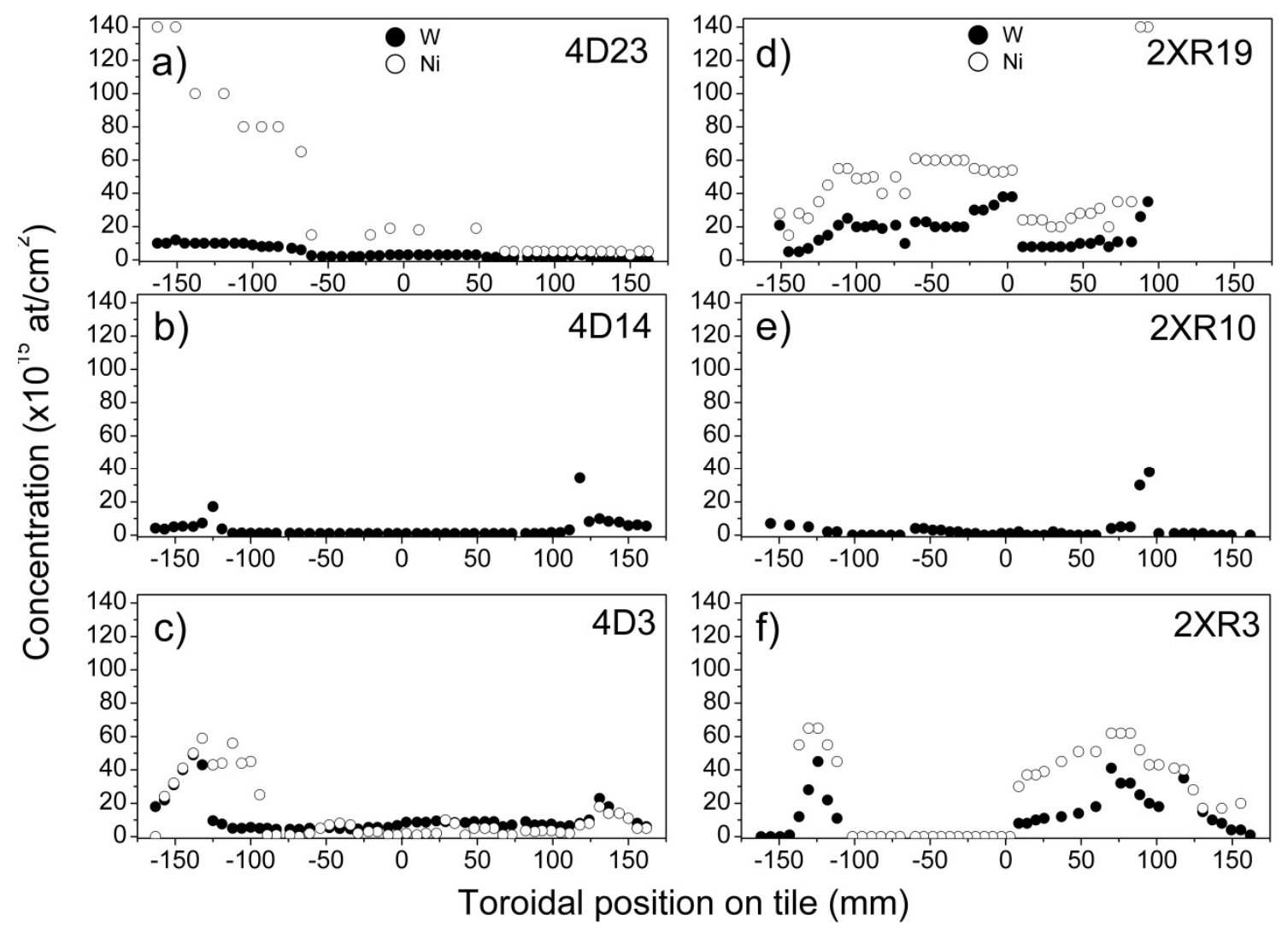

Fig. 3 


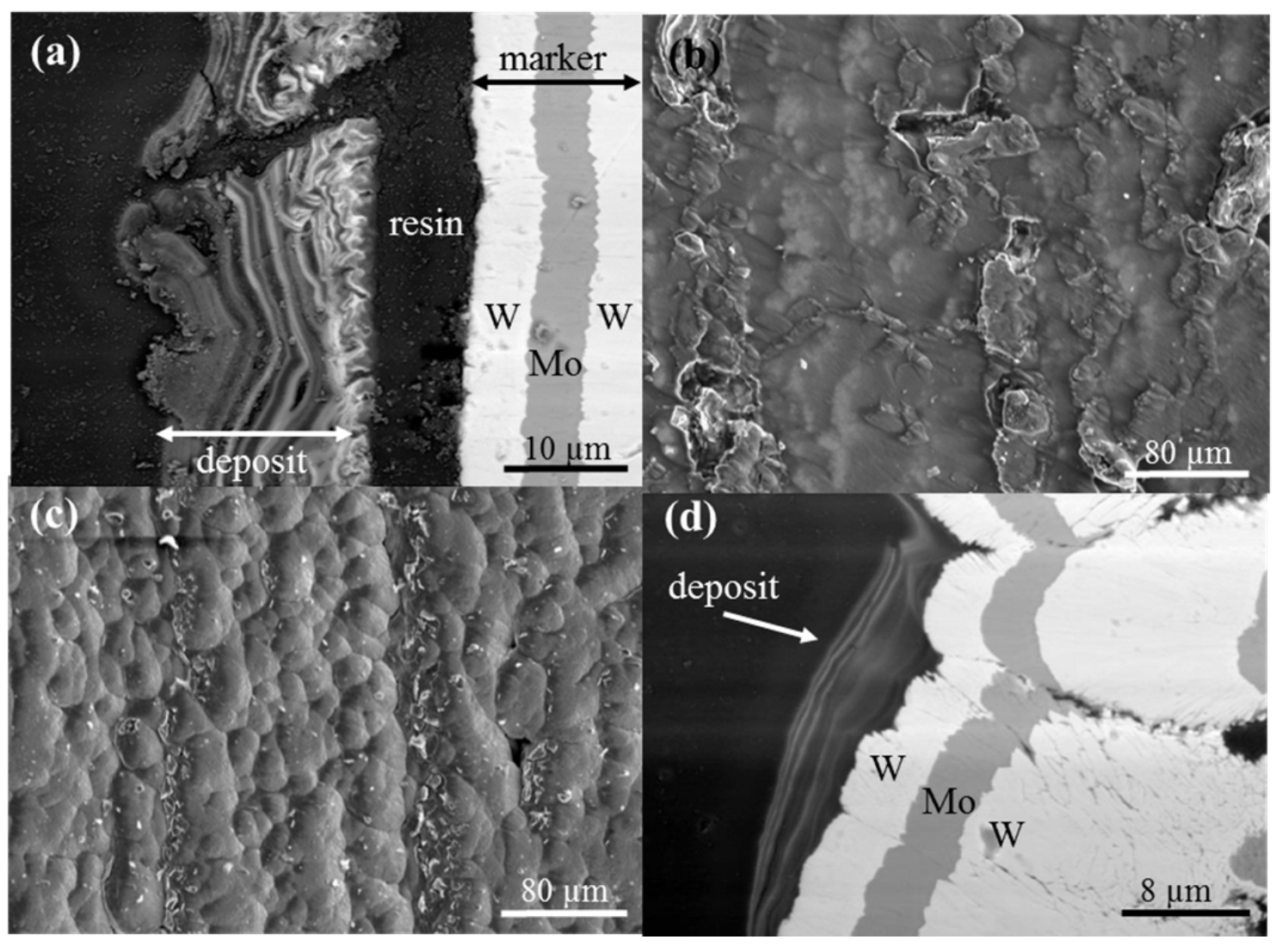

Fig.4. 
Table 1.

\begin{tabular}{|c|c|c|}
\hline & $\begin{array}{c}\text { JET-ILW }(2011-2) \\
\text { includes this work }[3,4]\end{array}$ & $\begin{array}{c}\text { JET-C (2007-2009) } \\
{[3]}\end{array}$ \\
\hline Plasma time, h (Limiter/Divertor) & $6 / 13$ & $12 / 33$ \\
\hline Dump Plates & "melting" & $130 \mathrm{~g}(-)$ \\
\hline \multicolumn{3}{|l|}{ IWGL } \\
\hline 2XR19 (1 row) & 0 & $0.8 \mathrm{~g}(+)$ \\
\hline 2XR10 (1 row) & $8 g(-)$ & $11 \mathrm{~g}(-)$ \\
\hline 2XR3 (1 row) & 0 & $\lg (+)$ \\
\hline IWC (all) & $15 \mathrm{~g}(-)[16]$ & $129 \mathrm{~g}(-)$ \\
\hline \multicolumn{3}{|l|}{ OPL } \\
\hline 4D23 (1 row) & 0 & 0 \\
\hline 4D14 (1 row) & $5 \mathrm{~g}(-)$ & $3.1 \mathrm{~g}(-)$ \\
\hline 4D3 (1 row) & 0 & $\lg (+)$ \\
\hline \multicolumn{3}{|l|}{ Divertor: } \\
\hline HFGC & $5-10(+)$ & $30 \mathrm{~g}(+)[17]$ \\
\hline Tile 1 & $25 \mathrm{~g}(+)$ & $65 \mathrm{~g}(+)[17]$ \\
\hline Tile $3,4,6$ & $<6 \mathrm{~g}(+)$ & $428 \mathrm{~g}(+)[17]$ \\
\hline Tile 7,8 & $<4 \mathrm{~g}(-)$ & $64 \mathrm{~g}(-)$ \\
\hline Dust & $1[3]$ & 233 \\
\hline
\end{tabular}

+ indicates deposition, - indicates erosion, 